\title{
GenCoNet: medical information system to support the treatment of co-morbid diseases
}

\author{
Ralf Hofestädt \\ University Bielefeld \\ AG Bioinformatics \\ Bielefeld, Germany
}

\author{
Elena Bragina \\ Tomsk NRMC \\ Institute of Medical Genetics \\ Tomsk, Russia
}

\author{
Victor Dosenko \\ National Academy of Sciences \\ Bogomoletz Institute of Physiolog \\ Kiev, Ukraine
}

\begin{abstract}
During the realization of the "Trilateral Partnership - Cooperation Project of the VolkswagenStiftung" between Scholars and Scientists from Ukraine (Kiev), Russia (Tomsk and Novosibirsk) and Germany (Bielefeld) (2016-2019) we have obtained important data and results concerning pathogenetic factors of such important comorbidity as combination of hypertension and asthma. Based on this project the medical information system GenCoNet was developed. During the last months an extended version of GenCoNet could be implemented.
\end{abstract}

Keywords - medical information system, co-morbid diseases, GenCoNet

\section{INTRODUCTION}

Hypertension and bronchial asthma are a major issue for people's health. As of 2014, approximately one billion adults, or $\sim 22 \%$ of the world population, have had hypertension. As of 2011, 235 - 330 million people globally have been affected by asthma and approximately 250,000 - 345,000 people have died each year from the disease. The development of the effective treatment therapies against these diseases is complicated by their comorbidity features.

Based on the medical data and knowledge of the project partner from Tomsk National Research Medical Center we could start to identify relevant genes and drugs for asthma and hypertension. Based on lists of genes associated with asthma and hypertension obtained using the HuGENavigator resource and patient drug lists, Bielefeld and Novosibirsk computed and analyzed first relevant metabolic networks. Finally, the project partner form Novosibirsk identified important genes that are involved in comorbid links between asthma and hypertension by using text- and data- mining techniques. The experimental analysis of the detected gene candidates showed that $I L 10$ silencing leads to decrease of severity of asthma and to positive changes in cardiohaemodynamic parameters. Furthermore, based on the clinical data and semi-automatic data mining approaches a new database was developed and implemented, which presents the positive and negative drug list for asthma and hypertension. A web based implementation of this data base allows the access to this information via internet (https://genconet.kalis-amts.de). A total of 126 subjects (42 healthy volunteers and 41 Crohn's disease and 43 ulcerative colitis patients) were studied. IBD was diagnosed on the basis of standard clinical, endoscopic, and histological criteria. Fecal microbiota composition was analyzed by $16 \mathrm{~S}$ and whole-genome shotgun sequencing.

\section{WORKFLOW}

The investigation of multifactorial diseases requires the consideration of the most important biomedical entities: diseases, genes, variants, and drugs. The data on these entities is spread across several data sources. These data sources need to be researched, identified, and integrated into a suitable database format. For this purpose, a workflow system was developed to extract, transform, and load reliable data on these entities into a uniform graph database eGenCoNet for network analysis of gene-disease associations. The workflow system is divided into four steps as illustrated in Figure 1.

First, the comorbidities of high interest in molecular medicine were determined. Essential hypertension and bronchial asthma are considered as an example of common comorbid diseases. Disease Ontology terms associated with genes were obtained from Osborne et al. Second, human genes that are associated with diseases and variants were obtained from Human Phenotype Ontology (HPO), MalaCards, DisGeNet, and OMIM. In particular, human genes were extracted from these databases that may cause familial syndromes (Mendelian forms). HPO and DisGeNet provide several database subsets from which the "FREQUENT FEATURES" marked data for HPO and "curated" marked data for DisGeNet were used. MalaCards does not provide a download of the database, so information was manually extracted and integrated from the website. From the OMIM gene map, information was extracted with the phenotype "Asthma, susceptibility to", "Hypertension, essential, susceptibility to" and adjacent annotation. In addition, altered expression data of genes associated with high blood pressure or severe asthma were manually curated and integrated.Third, genes that are controlled by eQTL in blood, codes variants, and gene associations supported by at least two studies were obtained from GWAS Catalog, dbSNP, and DisGeNet. These genetic variants were in turn associated with the comorbid diseases. Fourth, drugs and their target genes in humans were extracted from DrugBank using the full database XML version 5.1.0 including meta information like knownactions. Additional drugs, that are indicated, contraindicated or induced in asthma and hypertension were extracted from the DrugCentral 2017-08-29 database dump. 


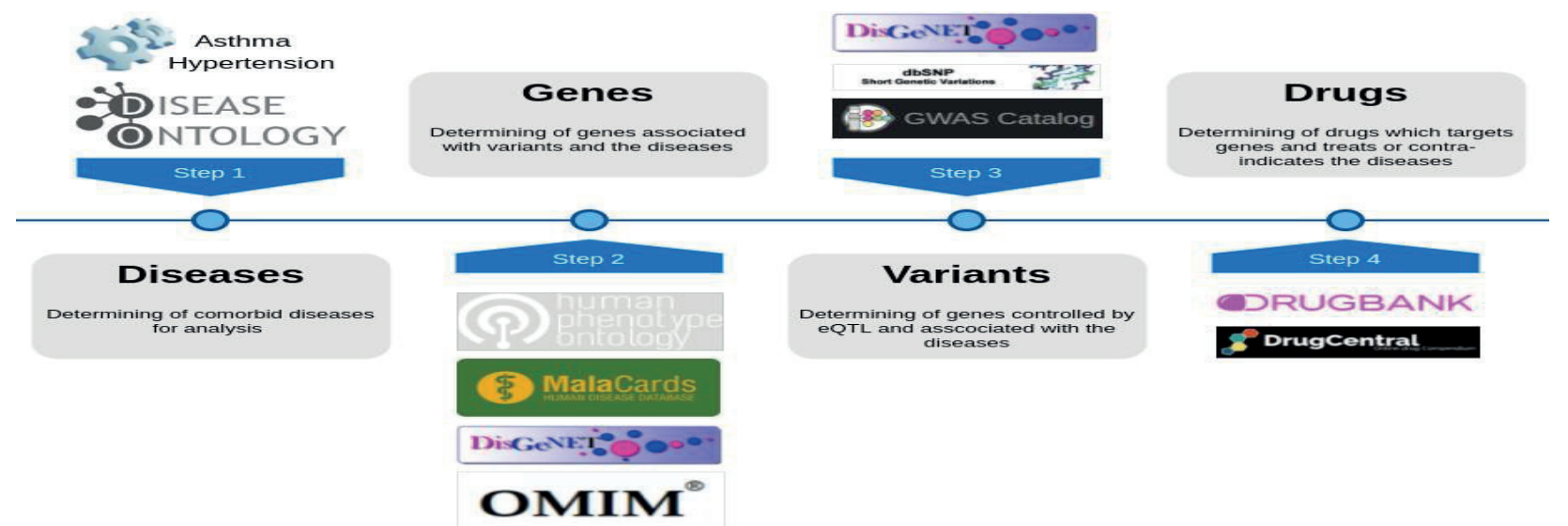

Fig. 1. Four steps of the workflow system for generating GenCoNet semi-automatically.

\section{IMPLEMENTATION}

The process of integrating all data sources and merging of the entities of the aforementioned workflow system is an iterative process. Therefore, a semi-automatic pipeline was implemented for the import, fusion, and analysis of data in a highly connected database structure. This pipeline provides custom and very fast import Python scripts and Cypher3 queries for generating a new Neo4j database from large data. TheNeo4j instance is running in a Docker4 container to simplify the setup process. Each step of the workflow system is executed in a separate Python script in order to have a better overview and to be able to execute specific stepson demand. Some of the data sources need to be preprocessed due to the higher information density or more complicated file formats than others. Afterwards, the data sources are processed and the basic connections are formed. Following nodes representing the same kind of entities are merged into fusion nodes. Finally, the results were imported into the new GenCoNet database

\section{APPICATION}

The prevalence of comorbidity is increasing and leads to a corresponding polypharmacy, which in turn is the prime risk factor for drug-related problems. In particular, drug contraindications with any disease and drug-induced diseases have to be considered in the context of treatment by healthcare professionals. Using GenCoNet, these risks may be detected (https://genconet.kalis-amts.de). The web interface represents the database entities (diseases, drugs, and proteins) as nodes in a network with edges representing the relationships between them. The vizualistion of networks show drugs (violet) which

are prescribed for the treatment of the comorbid diseases (green) but also target genes (red) which maycause the diseases. For instance, the anti-asthmatic drug "Ibudilast" may induce hypertension by targeting thegene PDE3A. As a consequence, these drugs have to be avoided in order to reduce the risk of induced diseases.

\section{DISCUSSION}

The discovery of shared molecular players and mechanisms in the pathogenesis of comorbid diseases is still complicated and nevertheless, necessary for decision-making of the most appropriate treatment strategy. To address this ongoing need, the Neo4j database GenCoNet was developed which integrates various associations between diseases, genes, variants and drugs for the diseases bronchial asthma and essential hypertension. While information of highest quality is preferred for integration in GenCoNet, false positives, although rare, cannot be ruled out. However, the use cases and lists already emphasized the potential of applicability in daily medical practice. GenCoNet is meant to be a qualitative resource that facilitates researchers access to the relevant information for the net-work analysis of comorbidities. Therefore, GenCoNet is planned to be extended by data on further diseases.

\section{REFERENCES}

[1] A. Shoshi, R. Hofestädt, O. Zolotareva, M. Friedrichs, A. Maier, V. A. Ivanisenko, V. E. Dosenko, E. Yu. Bragina. GenCoNet - A Graph Database for the Analysis of Comorbidities by Gene Networks. Journal of Integrative Bioinformatics 15(4), 2018. DOI: 10.1515/jib-20180049 . 